\title{
Macroinvertebrados dulceacuícolas del Parque Nacional Yendegaia, Chile: resolviendo brechas de conocimiento sobre la biodiversidad de la Reserva de la Biosfera Cabo de Hornos
}

\author{
Freshwater macroinvertebrates from Yendegaia National Park, Chile: \\ tackling knowledge biodiversity gaps in the Cape Horn Biosphere Reserve
}

Javier Rendoll-Cárcamo ${ }^{1,2,3,4}$, Melisa Gañán ${ }^{1,5}$, Roy Mackenzie ${ }^{1,2,3}$, Sophia Troncoso ${ }^{1}$, Javiera Troncoso ${ }^{1}$, Tamara Contador ${ }^{1,2,3,5}$, Ricardo Rozzi ${ }^{2,3,6}$ \& Peter Convey ${ }^{7}$

\section{Resumen}

El Parque Nacional Yendegaia, ubicado al sur de Tierra del Fuego y al margen de la vertiente oriental de la Cordillera Darwin, es parte de la Reserva de la Biosfera Cabo de Hornos, en la ecorregión subantártica de Magallanes. Este parque nacional del extremo austral de Chile comprende un extenso valle rodeado de cadenas montañosas con ríos de origen glaciar y pluvio-nivales. El presente estudio constituye el primer inventario de macroinvertebrados dulceacuícolas dentro del parque, y compara la composición de especies en tres ríos con bosques ribereños contrastantes. Un río tiene vegetación ribereña dominada coihüe de Magallanes (Nothofagus betuloides), una especie siempreverde. Los bosques ribereños de los otros dos ríos están dominados por especies arbóreas caducifolias, uno por ñirre $(N$. antarctica) y el otro por lenga ( $N$. pumilio). El río con vegetación ribereña dominada por lenga presentó una diversidad de macroinvertebrados significativamente mayor que en los ríos con vegetación ribereña dominada por ñirre y coihüe. La composición comunitaria de macroinvertebrados presenta algunas afinidades con la reportada para otras áreas dentro de la reserva con composición vegetacional similar, como Isla Navarino o el Parque Nacional Alberto de Agostini. La influencia de la vegetación ribereña y otras variables ambientales deben ser estudiadas a través de otros enfoques. De esta manera, emerge la necesidad de estudios que complementen el inventario aquí presentado, aportando a una caracterización que permita la evaluación de patrones ecológicos de los macroinvertebrados acuáticos. Es así, que se podrán determinar las generalidades y singularidades de las comunidades dulceacuícolas y sus relaciones ambientales en el extremo sur de Chile.

\section{Palabras Claves:}

Dulceacuícola, Ecología, ecorregión subantártica, Parques Nacionales, limnología.

\section{Abstract}

The Yendegaia National Park, located to the south of Tierra del Fuego and at the eastern margin of the Darwin Mountain Range, forms

Laboratorio Wankara de Ecosistemas Dulceacuícolas Subantárticos y Antárticos, Universidad de Magallanes \& Instituto de Ecología y Biodiversidad (IEB),

Puerto Williams, Chile

https://orcid.org/0000-0003-1928-0914

\javier.rendoll@gmail.com

2 Programa de Conservación Biocultural Subantártica, Universidad de Magallanes, Chile.

3 Instituto de Ecología y Biodiversidad, Estación de Campo Parque Omora,

Teniente Muñoz 166, Puerto Williams, Chile.

4 Programa de Doctorado en Ciencias Antárticas y Subantárticas, Universidad de Magallanes, Chile.

5 Núcleo Milenio de Salmónidos Invasores, INVASAL, Iniciativa Científica Milenio, Concepción, Chile.

6 Department of Philosophy and Religion Studies, University of North Texas, Denton, Texas, United States.

7 British Antarctic Survey, NERC,

Cambridge, CB3 OET, UK. 
part of the Cape Horn Biosphere Reserve (CHBR) and lies in the Magellanic sub-Antarctic ecoregion. This national park in the extreme south of Chile comprises an extensive valley surrounded by mountain ranges that give rise to glacial and rain or snow-melt streams. The present study provides the first inventory of freshwater macroinvertebrates within the park, further comparing the species composition of three rivers with contrasting riparian vegetation. One river has a riparian vegetation dominated by Coihüe de Magallanes (Nothofagus betuloides), an evergreen species. The river with riparian vegetation dominated by lenga presented a significantly higher diversity of macroinvertebrates than in the rivers with riparian vegetation dominated by ñirre and coihüe. The community composition of macroinvertebrates presents some affinities with that reported for other areas within the reserve with similar vegetational composition, such as Navarino Island or the Alberto de Agostini National Park. The influence of riparian vegetation and other environmental variables must be studied through other approaches. In this way, the need for studies that complement the inventory presented here emerges, contributing to a characterization that allows the evaluation of ecological patterns of aquatic macroinvertebrates. In this way, it will be possible to identify generalities and singularities of the freshwater biological communities and their environmental relationships in the southern tip of Chile.

\section{Key words:}

freshwater, Ecology, sub-Antarctic ecoregion, National Parks, Limnology.

\section{INTRODUCCIÓN}

Los invertebrados son los organismos multicelulares dominantes en términos de riqueza y abundancia, y corresponden al $80 \%$ de las especies descritas en el mundo (Collen et al. 2012). Habitan prácticamente todos los tipos de ecosistemas y hábitats del planeta, incluyendo ambientes marinos, terrestres, y dulceacuícolas. Representan un componente clave de la estructura y función de los ecosistemas, de los que dependen otros seres vivos incluidos los humanos. Sin embargo, el actual régimen y magnitud del impacto antropogénico sobre los ecosistemas representan una severa amenaza a la biodiversidad, especialmente para los invertebrados (SánchezBayo \& Wyckhuys, 2019, Rendoll et al. 2020a). El impacto humano no es homogéneo entre las sociedades ni entre los tipos de hábitats, y los ambientes dulceacuícolas se encuentran bajo un riesgo mayor (Collen et al. 2012). Consecuentemente, lugares prístinos y remotos como la Reserva de la Biosfera Cabo de Hornos $(\mathrm{RBCH})$ donde los impactos antropogénicos son todavía incipientes, son globalmente importantes para monitorear y conservar, para proteger sus comunidades biológicas, incluyendo los macroinvertebrados dulceacuícolas.

Se estima que alrededor de 126.000 especies de invertebrados habitan durante alguna fase de su ciclo de vida en hábitats dulceacuícolas, y que entre un 10 y un $15 \%$ se han extinguido o se encuentran amenazados de extinción (Vörösmarty et al. 2010, Reid et al. 2019, Dudgeon 2019). En Europa (Hallman et al. 2017) y algunos sitios en otros continentes (Sánchez-Bayo \& Wyckhuys 2019) se ha registrado una abrupta disminución en la abundancia de insectos, y se ha estimado que la degradación de hábitats y la contaminación podría llevar a la extinción hasta un $40 \%$ de las especies de insectos durante el siglo XXI. A su vez, aproximadamente un $40 \%$ de estos insectos correspondería a especies que utilizan hábitats acuáticos. Sin embargo, la identificación tanto de las principales causas de esta amenaza de extinción como de los grupos taxonómicos o funcionales más amenazados, se basa en estudios desarrollados casi exclusivamente en el hemisferio norte (Contador et al. 2012, Sánchez-Bayo \& Wyckhuys op. cit.). Lugares remotos y bien conservados del hemisferio sur, especialmente zonas de latitudes altas, representan un gran vacío de información. Además, estas regiones pueden ofrecer oportunidades para superar estas brechas y disminuir la incertidumbre de nuestras evaluaciones ambientales y predicciones, al mismo tiempo que permiten conservar la entomofauna (Contador et al. 2020). En este contexto, el extremo austral de la ecorregión subantártica de Magallanes con su heterogénea orografía, fiordos, canales y cuencas 
hidrográficas, donde se localiza la $\mathrm{RBCH}$, ofrece una oportunidad ideal para abordar estas brechas (Rendoll-Cárcamo et al. 2020a).

La ecorregión subantártica de Magallanes incluye los bosques y humedales más australes del planeta que por su condición remota han experimentado escaso impacto antropogénico, desarrollo urbano e industrial (Rozzi et al. 2012). Para esta ecorregión se ha descrito un alto nivel de endemismo para plantas vasculares, novasculares y vertebrados (Rozzi et al. 2006), pero un esfuerzo sistemático similar para la fauna de macroinvertebrados dulceacuícolas no se ha realizado (Contador et al. 2012). No obstante, la descripción de nuevas especies sugiere que los niveles de endemismo podrían ser similares (Valdovinos et al. 2010, Morrone, 2015). La $\mathrm{RBCH}$ fue creada en 2005 para proteger el extremo austral de esta ecorregión archipelágica chilena, considerando un enfoque de conservación biocultural que incluye los ecosistemas terrestres, marinos, dulceacuícolas y sus interrelaciones (Rozzi et al. 2006). La RBCH es la reserva más grande de Chile, con 4.884.273 ha entre tierra (1.917.237 ha) y áreas marinas (2.967.036 ha). Al momento de la creación del $\mathrm{RBCH}$ no se había creado aún el Parque Nacional Yendegaia. Actualmente, junto a los parques nacionales Cabo de Hornos y Alberto de Agostini, es uno de los tres parques inmersos en la $\mathrm{RBCH}$ y en conjunto presentan atributos singulares (Rozzi et al. 2012). Este parque nacional está ubicado al suroeste de Tierra del Fuego y su extremo sur se conecta con el canal Beagle.

El PN Yendegaia, creado en 2013, se localiza sobre la vertiente oriental de la Cordillera Darwin y protege extensos valles glaciares. En su sector sur, nace del glaciar Stoppani el mayor río del PN, el río Yendegaia. Sin embargo, a lo largo de todas las laderas nacen centenares de arroyos que fluyen desde los glaciares y el derretimiento de nieve acumulada en invierno en las cumbres montañosas. Este parque aún no cuenta con una caracterización físico-química ni biótica para los ecosistemas dulceacuícolas. La fauna dulceacuícola de la $\mathrm{RBCH}$ se encuentra directamente influenciada por marcados gradientes ambientales, principalmente por agudos cambios en la temperatura en perfiles de elevación (Contador et al. 2015, RendollCárcamo et al. 2019). Sin embargo, la estructura de estas comunidades de macroinvertebrados ha sido evaluada en cuencas de origen pluvionival y son escasos los estudios en ambientes con influencia glaciar (Moorman et al. 2006). La influencia directa del valle glaciar limita las extrapolaciones desde otras áreas estudiadas dentro de la reserva.

La vegetación ribereña constituye un ecotono entre los hábitats terrestre y acuático, con características bióticas y físicas únicas que son determinantes en la estructura y funcionalidad de los cuerpos de agua (Mancilla et al. 2009, Simanonok et al. 2011). Además, constituye la principal fuente de aportes energéticos a los ríos y alimento para los macroinvertebrados dulceacuícolas (Vannote et al. 1980). No obstante, su relevancia se encuentra altamente impactada por el desarrollo de diversas actividades antrópicas (Dudgeon, 2019). En este contexto, una línea base sobre la diversidad local es un importante primer paso para el desarrollo de preguntas de investigación más complejas. Asimismo, es necesario generar información que permita el diseño e implementación de estrategias para el manejo y conservación de valles glaciares, uno de los ecosistemas más vulnerables al cambio ambiental global (Reid et al. 2018, Dudgeon, 2019).

A pesar de su ubicación remota, el PN Yendegaia tendrá un cambio en su conectividad dentro de esta década debido a la construcción de la carretera Yendegaia - Vicuña que conectará el sur de Tierra del Fuego con el continente (Sernatur, 2014). Esta ruta puede traer beneficios económicos al sector turístico si se administra adecuadamente. Sin embargo, para que ello ocurra es crítico monitorear y evaluar los potenciales efectos de las actividades que se desarrollarán en el lugar, con la finalidad de evitar la entrada de especies exóticas o la degradación de los hábitats (Schüttler et al. 2019).

En este contexto, los objetivos del presente trabajo son: i) determinar la riqueza, abundancia y diversidad de macroinvertebrados en tres ríos contrastantes del PN Yendegaia (RBCH); ii) evaluar la variabilidad de los ensambles comunitarios de macroinvertebrados en relación a variables ambientales en estos ríos. 


\section{MATERIALES Y MÉTODOS}

\section{Área de estudio}

La ecorregión subantártica de Magallanes se caracteriza por la presencia de cuencas hidrográficas con agudos gradientes ambientales, marcados perfiles de vegetación y una amplia variedad de hábitats y microhábitats en una corta elevación (Contador et al. 2015, Rendoll-Cárcamo et al. 2019). Debido a su ubicación geográfica fuera de las corrientes atmosféricas que acarrean contaminantes industriales, las aguas de lluvia, los cuerpos de agua presentan una de las concentraciones más bajas de contaminantes inorgánicos registradas en el planeta (Hedin et al. 1995, Mach et al. 2016). La RBCH es parte de esta ecorregión y refuerza la conservación de sus ecosistemas más australes (RBCH; Fig. 1).

Sitios de estudio, caracterización ambiental y parámetros físico-químicos

En el Parque Nacional Yendegaia existen numerosos ríos que se pueden agrupar bajo dos categorías, glaciares y pluvio-nivales. En el presente estudio nos enfocamos en los últimos.
Los tres ríos pluvio-nivales relevados (Fig. 1) se encuentran rodeados por vegetación ribereña dominada por especies diferentes del género Nothofagus: N. antarctica (río Nirre), N. pumilio (río Lenga), especies caducifolias, y $N$. betuloides (río Coihüe), especie siempreverde. Cercano al río Nirre se encuentra un asentamiento humano, principalmente Carabineros y Cuerpo Militar del Trabajo. Además, existe ganado bovino y extracción de leña en los alrededores del río. El río Lenga es cruzado por el camino en construcción y tiene un alcantarillado que resguarda su integridad. En cada río, y debido a su accesibilidad, se identificaron cuatro sitios para la caracterización ambiental y colecta de macroinvertebrados durante otoño (abril) de 2017. La elección de estos sitios se limitó a los tiempos de la campaña de inventario, manteniendo una separación de $\sim 250$ m entre cada sitio. Para la caracterización fisicoquímica (Tabla 1, S1) de cada sitio se consideró el tipo de substrato, presencia/ ausencia de macrófitas acuáticos, presencia/ ausencia de briófitas acuáticas o sumergidas, y profundidad. Tanto las macrófitas como las briófitas no fueron incluidas en los análisis debido a que no se realizaron mediciones cuantitativas. Adicionalmente, se registró temperatura, $\mathrm{pH}$,

Tabla 1. Parámetros abióticos y métricas de diversidad. Valores promedio ( \pm error estándar) y resultados del análisis no paramétrico de Kruskal-Wallis para los parámetros abióticos y métricas de diversidad registradas en ríos pluvio-nivales del

Parque Nacional Yendegaia, sur de Chile. Abreviaciones: OD = oxígeno disuelto, SDT = sólidos disueltos totales. Los valores de $p$ en negrita denotan diferencias significativas $(p<0,05)$.

\begin{tabular}{|c|c|c|c|c|c|c|}
\hline & & Nirre & Lenga & Coihüe & $\mathrm{H}$ & $p$ \\
\hline \multirow[t]{6}{*}{$\begin{array}{c}\text { PARÁMETROS } \\
\text { ABIÓTICOS }\end{array}$} & $\begin{array}{c}\text { Temperatura } \\
\left({ }^{\circ} \mathrm{C}\right)\end{array}$ & $5,79( \pm 0,33)$ & $2,87( \pm 0,34)$ & $4,98( \pm 0,35)$ & 30,126 & 0,00001 \\
\hline & $\mathrm{pH}$ & $7,92( \pm 0,11)$ & $7,69( \pm 0,06)$ & $7,35( \pm 0,05)$ & 30,725 & 0,00001 \\
\hline & $\begin{array}{c}\text { Conductividad } \\
(\mu \mathrm{S} / \mathrm{cm})\end{array}$ & $55,38( \pm 2,49)$ & $54,65( \pm 6,63)$ & $29,53( \pm 5,36)$ & 24,052 & 0,00001 \\
\hline & $\mathrm{OD}(\%)$ & $73,06( \pm 5,18)$ & $88,17( \pm 5,84)$ & $96,87( \pm 1,29)$ & 28,417 & 0,00001 \\
\hline & $\mathrm{SDT}(\mathrm{mg} / \mathrm{L})$ & $0,07( \pm 0,01)$ & $0,06( \pm 0,01)$ & $0,07( \pm 0,01)$ & 1,225 & 0,542 \\
\hline & $\begin{array}{l}\text { Profundidad } \\
\text { (cm) }\end{array}$ & $10,63( \pm 2,24)$ & $12,84( \pm 3,36)$ & $16,52( \pm 8,84)$ & 5,162 & 0,075 \\
\hline \multirow[t]{5}{*}{$\begin{array}{l}\text { MÉTRICAS DE } \\
\text { DIVERSIDAD }\end{array}$} & Riqueza (S) & $10( \pm 0,41)$ & $10,67( \pm 0,74)$ & $6,33( \pm 0,40)$ & 18,64 & 0,00001 \\
\hline & $\begin{array}{l}\text { Abundancia } \\
\text { (N) }\end{array}$ & $258,42( \pm 31,04)$ & $59,92( \pm 14,72)$ & $18,08( \pm 2,62)$ & 28,83 & 0,00001 \\
\hline & $\begin{array}{l}\text { Equitatividad } \\
\text { de Pielou }\left(J^{\prime}\right)\end{array}$ & $0,55( \pm 0,03)$ & $0,82( \pm 0,02)$ & $0,80( \pm 0,02)$ & 18,70 & 0,00001 \\
\hline & N1 de Hill & $3,61( \pm 0,31)$ & $7,02( \pm 0,49)$ & $4,41( \pm 0,31)$ & 20,51 & 0,00001 \\
\hline & N2 de Hill & $2,59( \pm 0,22)$ & $5,58( \pm 0,49)$ & $3,39( \pm 0,28)$ & 20,47 & 0,00001 \\
\hline
\end{tabular}




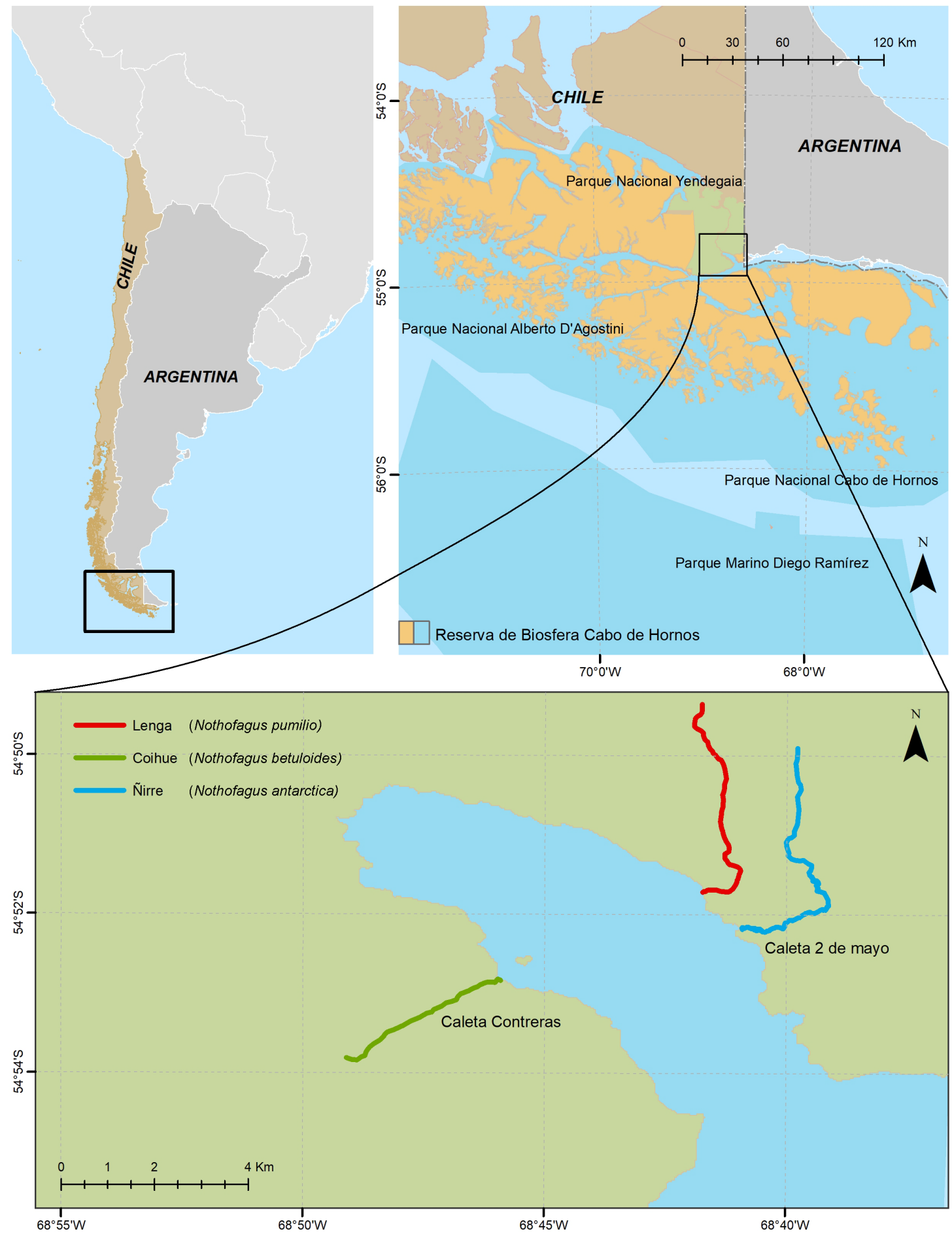

Fig. 1. Reserva de la Biosfera Cabo de Hornos y sitios de estudio en el Parque Nacional Yendegaia. Ubicación de la Reserva de la Biosfera Cabo de Hornos en el Sur de Chile y los ríos pluvio-nivales estudiados (diferenciados por color según su vegetación ribereña dominante) dentro del Parque Nacional Yendegaia en la figura aumentada (SRC WGS84, elaboración propia, QGIS 3.8.1. Zanzibar). 
conductividad, sólidos disueltos totales y oxígeno disuelto en cada sitio (tres mediciones por sitio) utilizando sensores de multiparámetro (YSI 605 595 Professional Plus multimeter y ConductivitypH-TDS Hanna Tester HI98130).

\section{Colecta de macroinvertebrados e identificación}

La colecta tuvo lugar en abril de 2017. Para cada río se seleccionaron cuatro sitios de colecta definidos por la accesibilidad del mismo, y en cada uno se tomaron tres muestras con red Surber (malla de $243 \mu \mathrm{m}$ y $0,09 \mathrm{~m}^{2}$ de área). Los macroinvertebrados fueron almacenados en frascos con alcohol al $90 \%$, y posteriormente transportados a laboratorio para su identificación. Para las identificaciones taxonómicas se siguió la literatura pertinente disponible para cada grupo (e.g. Domínguez \& Fernández, 2009, Flint et al. 1999, McLellan \& Swick, 2007). Los taxa identificados se clasificaron en grupos funcionales de alimentación (GFA: trituradores, depredadores, colectores, raspadores y filtradores), según Merritt \& Cummins (2008), Domínguez y Fernández (2009) y Contador et al. (2015). Los permisos de campo y colecta fueron otorgados por la Corporación Nacional Forestal en el marco del proyecto CORFO BPER16.

\section{Análisis de los datos}

Los datos fisicoquímicos ( $\mathrm{n}=12$, tres réplicas para cada sitio en los tres ríos) presentaron una distribución no-paramétrica (prueba de ShapiroWilks). En consecuencia, se realizó un análisis de Kruskal-Wallis de una vía y comparaciones a posteriori de Wilcoxon para evaluar las diferencias en los parámetros abióticos entre los ríos estudiados. Las métricas e índices de diversidad calculados para cada río fueron: riqueza de especies, abundancia, equitatividad de Pielou $\left(\mathrm{J}^{\top}\right)$, y los números de Hill N1 y N2 (Chao et al. 2014). Éstos últimos representan un equivalente a los indices de diversidad de Shannon (N1) y Simpson (N2), sin embargo, la unidad "número efectivo de especies" de este análisis, permite estandarizar la comparación de la estructura comunitaria. La abundancia de macroinvertebrados de cada río se presenta como el promedio de las réplicas en cada sitio. Al igual que para los parámetros abióticos, el análisis de Kruskal-Wallis y las comparaciones a posteriori de Wilcoxon fueron calculadas para evidenciar diferencias significativas entre las métricas de diversidad obtenidas para cada río. Para evaluar los cambios estructurales de la diversidad de macroinvertebrados en los ríos estudiados, se realizó el análisis por permutaciones PERMANOVA (Anderson, 2001), posterior a la prueba PERMDISP para evaluar la homogeneidad de éstos, dentro y entre los grupos (Anderson, 2005). La prueba PERMANOVA sólo asume que las muestras son intercambiables bajo una verdadera hipótesis nula. La suposición de intercambiabilidad equivale al supuesto de que las muestras son independientes y están distribuidas de manera idéntica (Anderson et al. 2008). Aunque no existen supuestos explícitos sobre las distribuciones de las variables originales, la independencia y homogeneidad de las dispersiones están directamente implícitas en el procedimiento de permutación. Además, si las muestras están correlacionadas de forma desigual entre sí (temporal o espacialmente), mezclarlas aleatoriamente destruirá este tipo de estructura inherente (Anderson et al. 2008). Cuando se realizan permutaciones, los valores de las diferentes variables dentro de una muestra se mantienen como unidad, por lo que cualquier estructura de correlación no se altera con la permutación. Para el PERMANOVA, se consideraron dos factores, los ríos (factor fijo) y los sitios (factor aleatorio), este último anidado dentro de los ríos. Adicionalmente, se utilizó el análisis de escalamiento multidimensional nométrico (nMDS) para la visualización de las similitudes o disimilitudes entre las comunidades de macroinvertebrados dulceacuícolas (Anderson et al. op. cit.). Los datos biológicos utilizados en el PERMANOVA y nMDS fueron estandarizados y transformados a raíz cuadrada previamente a la construcción de la matriz de similitud de Bray-Curtis. El análisis no paramétrico de Modelo Linear Basado en Distancias (DISTLM) fue utilizado en combinación con al Análisis de Ordenación de Redundancia Basado en Distancias (dbRDA) para explorar la hipótesis de que la variabilidad de los ensambles comunitarios puede ser explicada a través de variables ambientales. 
El análisis DISTLM modela la relación entre variables ambientales y los datos de abundancias del ensamble de macroinvertebrados a través de un modelo de regresiones múltiples. Este procedimiento utiliza la rutina BEST de Primer 7 para la elección del mejor procedimiento en conjunto con el criterio estadístico. El producto generado por BEST utilizado por DISTLM es el mejor modelo basado en una, dos, o más variables, de acuerdo con el criterio seleccionado; en este caso, el $\mathrm{R}^{2}$ ajustado (Anderson et al. op. cit., Clarke \& Gorley, 2015). Las rutinas DISTLM y dbRDA en conjunto buscan la mejor combinación de variables que explican la mayor cantidad de variación en un set de datos biológicos y examina la influencia de variables ambientales que explican esta varianza, proveyendo un valor estadístico de pseudo-F (Anderson et al. 2008). Los resultados de DISTLM son visualizados en un espacio multidimensional a través de la ordenación entregada por el dbRDA. El procedimiento dbRDA permite el trabajo con matrices basadas en distancias no Euclidianas para datos biológicos (distancia de Bray-Curtis), para posteriormente entregar un set de coordenadas que representan los resultados en un espacio Euclidiano (Anderson et al. 2008). Para determinar la importancia específica de macroinvertebrados en cada río y su grupo funcional asociado, se realizó el análisis de Porcentajes de Similitud SIMPER (Clarke, 1993), con un porcentaje de contribución al $80 \%$ para una mejor representatividad y posterior visualización. Los análisis fueron realizados en los softwares STATISTICA 7 (StatSoft Inc. 2004) y Primer 7 (PRIMER-E, Ltd. Plymouth, UK).

\section{RESULTADOS}

\section{Parámetros físico-químicos}

Los parámetros físico-químicos medidos en los tres ríos de régimen pluvio-nival presentaron diferencias significativas en la mayoría de sus mediciones (Tabla 1). Los registros de temperatura indicaron una variación significativa entre los ríos $(\mathrm{K}-\mathrm{W}, p<0,001)$. Estas diferencias se acentúan en el río Lenga con un promedio de $2,87^{\circ} \mathrm{C}$, siendo la temperatura más baja entre los ríos. Los ríos Nirre y Coihüe también presentaron diferencias significativas en los registros de temperatura $(5,79$ ${ }^{\circ} \mathrm{C}$ y $4,98{ }^{\circ} \mathrm{C}$, respectivamente), sin embargo, no tan acentuadas en relación con el río Lenga. Las medidas de $\mathrm{pH}$ también indicaron diferencias entre los ríos ( $\mathrm{K}-\mathrm{W}, p<0,001)$, no obstante, todos los valores registrados se encuentran cercanos a neutro. Adicionalmente, también presentaron diferencias significativas las medidas de conductividad $y$ oxígeno disuelto (OD \%) registrándose las mayores medidas de conductividad en el río Nirre $(55,38$ $\mu \mathrm{S} / \mathrm{cm})$ y las menores en río Coihüe $(29,53 \mu \mathrm{S} /$ $\mathrm{cm})$. Inversamente, en el río Coihüe se registraron los valores más altos de saturación de oxígeno y las menores en el río Nirre $(96,87$ \% y 73,06 $\%$, para el río Coihüe y Nirre respectivamente). La conductividad registrada en el río Coihüe fue significativamente más baja en comparación a la registrada en los ríos Nirre y Lenga; sin embargo, estos no presentaron diferencias significativas entre ellos (Tabla S2). Por último, las variaciones de sólidos disueltos totales (SDT) y profundidad no presentaron diferencias significativas (ver Tabla 1 y S2).

\section{Métricas de diversidad}

Un total de 4037 macroinvertebrados fueron colectados en el presente estudio, los que fueron clasificados en 23 taxa, nueve de ellos identificados a nivel de especies, ocho en géneros y siete familias o subfamilias, pertenecientes a ocho órdenes (Tabla 2). El río Lenga presentó la mayor riqueza absoluta $(\mathrm{S}=21)$ y el río Coihüe la menor $(\mathrm{S}=12)$. La riqueza promedio de los ríos Lenga y Nirre fue significativamente más alta que en el río Coihüe (Fig. 2). La abundancia de macroinvertebrados también presentó diferencias significativas entre todos los ríos (Tabla $1, \mathrm{~S} 3$ ), registrándose el valor más alto en el río Nirre $(\mathrm{N}=258,42)$ y el menor en el río Coihüe $(\mathrm{N}=18,08)$ (Fig. 2). Los índices de diversidad N1 y N2 de Hill también presentaron diferencias significativas entre los ríos, con los valores más altos en el río Lenga y los más bajos en el río Nirre (Tabla S3, Fig. 2). El índice de equitatividad de Pielou (J') reporta una variación significativa (Tabla 1) entre el río Nirre respecto a los otros dos ríos, indicando una comunidad con riqueza y abundancias más homogéneas en los ríos Lenga y Coihüe. 
Tabla 2. Lista taxonómica de macroinvertebrados asociados a tres ríos diferentes del presente estudio. Abreviaciones: GFA = grupos funcionales de alimentación, Col = colector, Fil = filtrador, Pred = depredador, Rasp $=$ raspador, Trit $=$ triturador. La "x" significa presencia en un río particular.

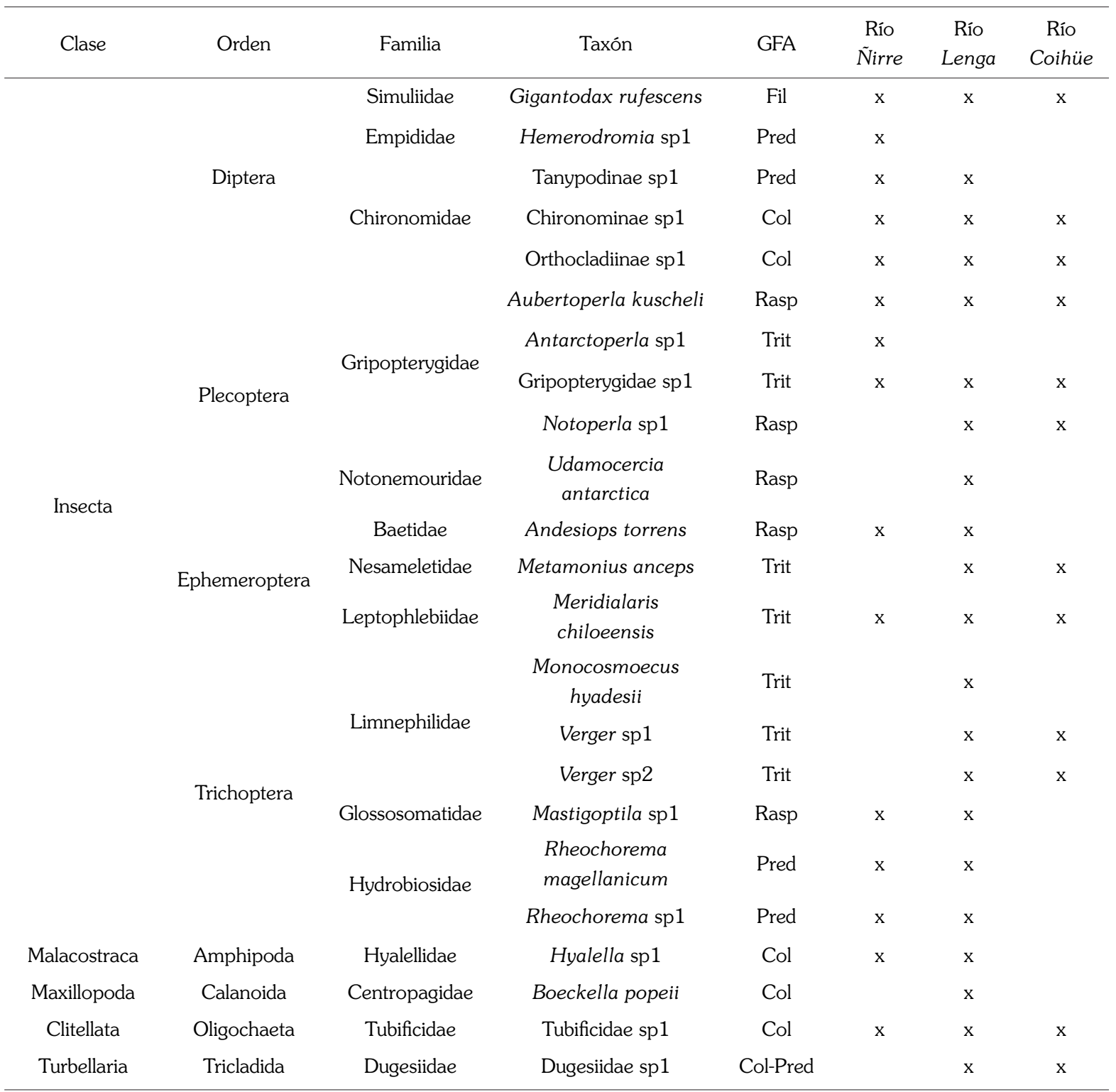

\section{Composición comunitaria}

Se detectaron diferencias significativas en la composición comunitaria entre los ríos, pero no entre los sitios de un mismo río (PERMANOVA $p=0,0003$ y $p=0,6234$, respectivamente; ver Tabla S4). El análisis nMDS muestra tres grupos principales respecto a la estructura comunitaria coincidente con los tres ríos (similitud 40\%, Fig.
3). El procedimiento BEST y el modelo linear basado en distancias (DISTLM) sugieren que cinco variables ambientales (temperatura, $\mathrm{pH}$, conductividad, oxígeno disuelto y profundidad) explican de mejor manera la varianza entre las comunidades de macroinvertebrados. La visualización de estos resultados en el dbRDA, muestra la influencia de estas variables ambientales, agrupando las comunidades propias de cada río $\left(\mathrm{R}^{2}\right.$ 


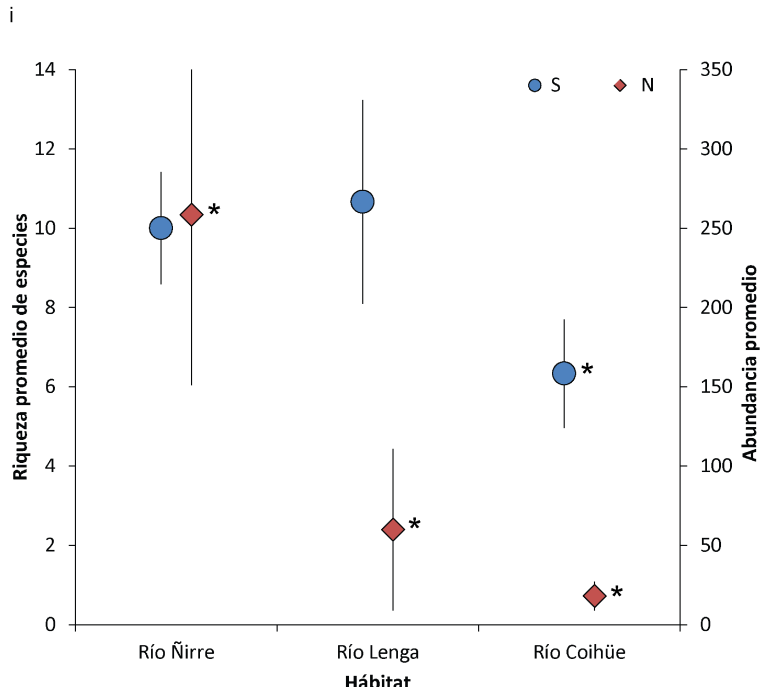

ii

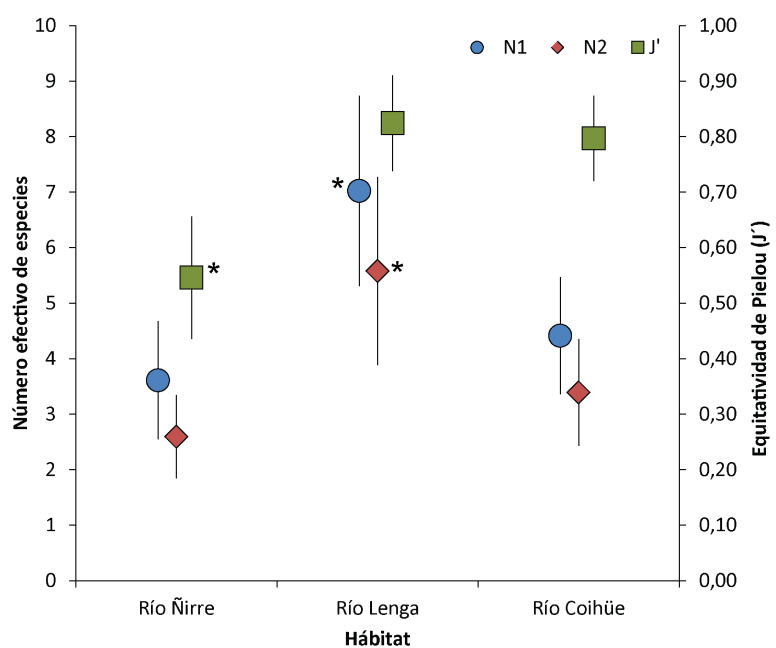

Fig. 2. i) Riqueza y abundancia promedio, ii) índices de diversidad de macroinvertebrados dulceacuícolas presentes en ríos del Parque Nacional Yendegaia, sur de Chile. Se reportan los valores de riqueza, abundancia e índices de diversidad de Hill ( \pm desviación estándar) para los tres ríos estudiados durante 2017. Los valores se reportan estandarizados y transformados a raíz cuadrada. Los asteriscos representan diferencias significativas ( $p<0,05)$ (detalles en Tabla S3).

ajustado=0,58). La mayor cantidad de variación en los datos de ensamble comunitario se encuentra explicada por la profundidad y temperatura, $y$ estas cinco variables en conjunto (Tabla 3, Fig. 4). Los ensambles de macroinvertebrados dulceacuícolas presentan disimilitudes en términos de composición, reflejadas en la importancia relativa de taxa particulares en cada río (Tabla 4, Fig. 5). Para el río Nirre se identificaron cinco taxa característicos; Aubertoperla kuscheli, Gigantodax rufescens, Meridialaris chiloeensis, quironómidos de la sub-familia Orthocladiinae y Andesiops torrens. Para la comunidad de macroinvertebrados del río Coihüe también se identificaron cinco taxa representativos; $M$. chiloeensis como la especie más abundante, gusanos tubifícidos, dos morfotipos del género Verger y el plecóptero Notoperla sp. Finalmente, el ensamble comunitario del río Lenga presentó ocho taxa conspicuos; quironómidos de la subfamilia Orthocladiinae y gusanos tubifícidos como los grupos más abundantes, los plecópteros A. kuscheli y Notoperla sp., los efímeros A. torrens y $M$. chiloeensis, anfípodos del género Hyalella y representantes de la subfamilia Chironominae.

Tabla 3. Valores de estadísticos obtenidos del modelo linear basado en distancias (DISTLM) para las variables ambientales identificadas por el procedimiento BEST $\left(\mathrm{R}^{2}=0,58\right)$.

Estas variables explican el mejor ajuste de la variación en la composición del ensamble de macroinvertebrados dulceacuícolas en ríos pluvio-nivales del Parque Nacional Yendegaia, sur de Chile. $\mathrm{OD}=$ oxígeno disuelto, SDT = sólidos disueltos totales. Los valores reportados de $p$ en negrita denotan variables significativas.

\begin{tabular}{cccc}
\hline Variable ambiental & Pseudo-F & $p$ & Proporción \\
\hline Temperatura & 6,472 & 0,001 & 0,160 \\
pH & 24,361 & 0,001 & 0,417 \\
Conductividad & 20,091 & 0,001 & 0,371 \\
OD & 22,373 & 0,001 & 0,397 \\
Profundidad & 3,419 & 0,008 & 0,091 \\
SDT & 1,422 & 0,193 & 0,040 \\
\hline
\end{tabular}


Fig. 3. Escalamiento multidimensional no-métrico (nMDS) de la composición de macroinvertebrados dulceacuícolas en ríos del Parque Nacional Yendegaia, sur de Chile. Las figuras geométricas dentro de los óvalos representan la fauna asociada con cada río. La línea negra denota una similitud

de $40 \%$ en la composición del ensamble de macroinvertebrados dulceacuícolas entre los ríos.

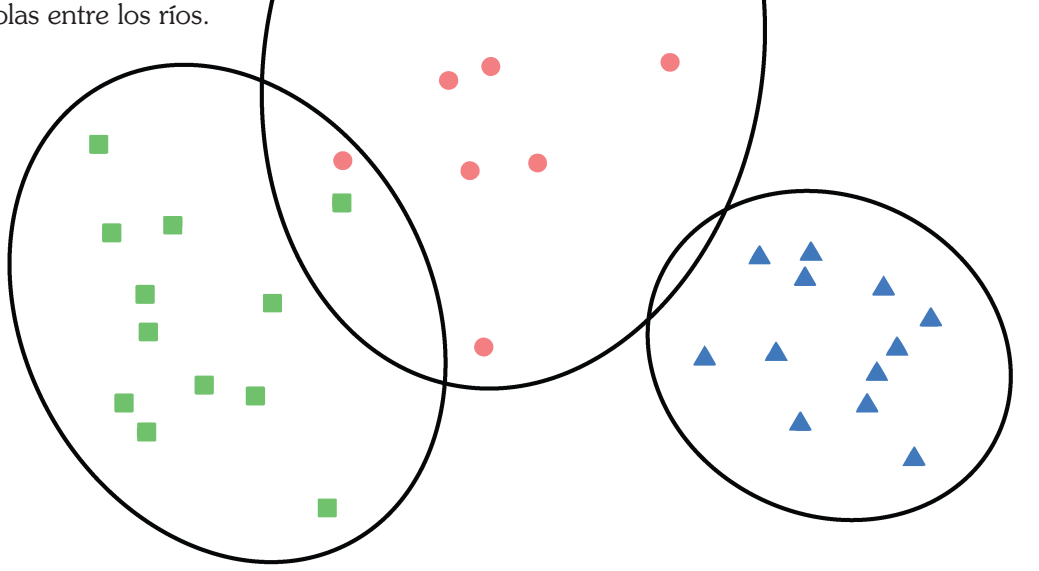

Tabla 4. Composición comunitaria de macroinvertebrados dulceacuícolas presentes en el Parque Nacional Yendegaia. Valores del análisis de porcentajes de similitud (SIMPER) de la composición del ensamble de macroinvertebrados dulceacuícolas en ríos pluvio-nivales del Parque Nacional Yendegaia, sur de Chile. El porcentaje de corte de contribución se fijó en $80 \%$ de la abundancia del ensamble. Av. Abund = Abundancia promedio, Sim/SD = Desviación estándar, Contrib\% = Contribución,

Cum\% = Valores de contribución acumulados. Los datos están estandarizados y transformados a raíz cuadrada.

\begin{tabular}{cccccc}
\hline Río & Especie/ morfoespecie & Av.Abund & Sim/SD & Contrib\% & Cum.\% \\
\hline \multirow{4}{*}{ Nirre } & Aubertoperla kuscheli & 6,38 & 3,59 & 32,55 & 32,55 \\
& Gigantodax rufescens & 5,9 & 2,57 & 28,97 & 61,52 \\
& Meridialaris chiloeensis & 2,26 & 2,81 & 9,98 & 71,5 \\
& Orthocladiinae sp1 & 1,67 & 2,67 & 7,23 & 78,73 \\
& Andesiops torrens & 1,42 & 1,31 & 5,37 & 84,1 \\
\hline \multirow{4}{*}{ Lenga } & Orthocladiinae sp1 & 3,76 & 1,45 & 17,23 & 17,23 \\
& Tubificidae sp1 & 3,78 & 1,39 & 16,96 & 34,19 \\
& Notoperla sp1 & 2,01 & 2,01 & 10,16 & 44,34 \\
& Andesiops torrens & 2,27 & 1,17 & 8,85 & 53,19 \\
& Hyalella sp1 & 2,46 & 1,1 & 8,85 & 62,04 \\
& Aubertoperla kuscheli & 2,02 & 1,27 & 8,68 & 70,72 \\
& Meridialaris chiloeensis & 2,43 & 0,64 & 7,32 & 78,04 \\
& Chironominae sp1 & 1,29 & 0,65 & 3,81 & 81,85 \\
\hline \multirow{4}{*}{ Coihüe } & Meridialaris chiloeensis & 6,62 & 3,43 & 40,59 & 40,59 \\
& Tubificidae sp1 & 3,7 & 2,02 & 19,13 & 59,72 \\
& Verger sp1 & 2,19 & 1,35 & 10,47 & 70,2 \\
& Verger sp2 & 2,11 & 1,07 & 9,37 & 79,57 \\
& Notoperla sp1 & 2,15 & 0,66 & 6,86 & 86,43 \\
\hline
\end{tabular}




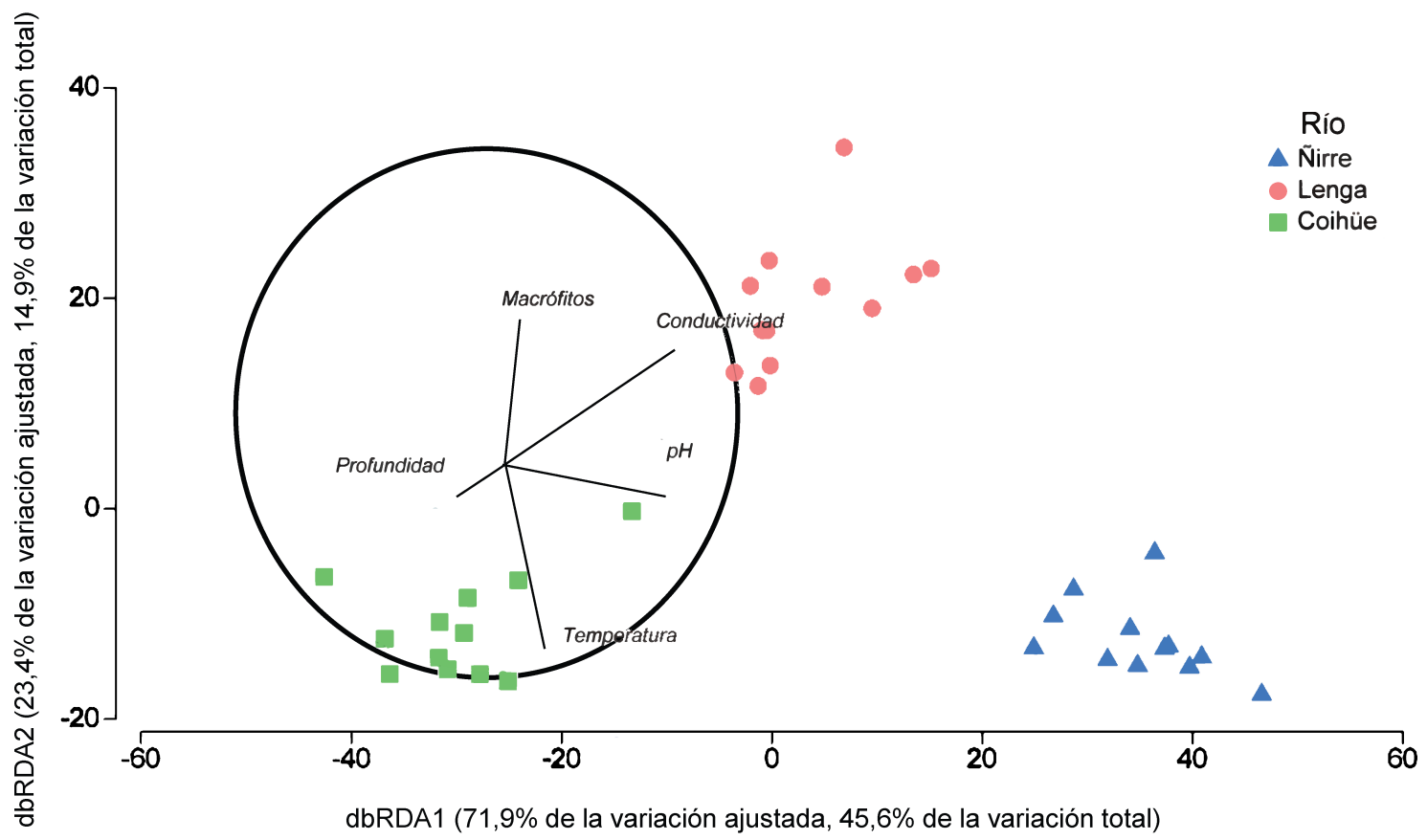

Fig. 4. Análisis de ordenación de redundancia basado en distancias (dbRDA) ilustrando la relación entre las variables ambientales predictoras que explican la variación de los ensambles de macroinvertebrados dulceacuícolas presentes en ríos del Parque Nacional Yendegaia, sur de Chile. El dbRDA presenta las variables explicativas (vectores) de mejor ajuste del análisis de regresión múltiple multivariado DISTLM.

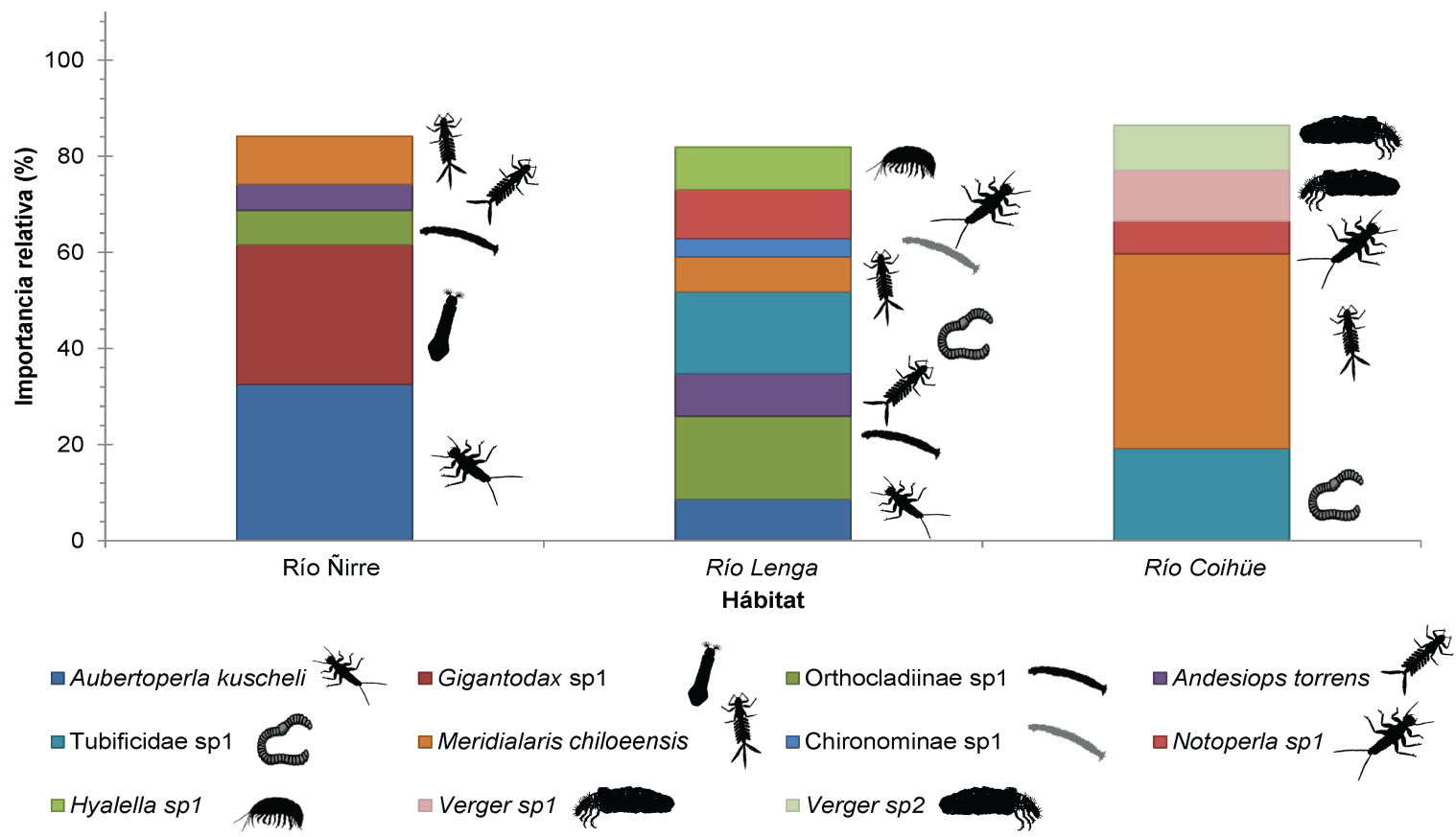

Fig. 5. Composición comunitaria de macroinvertebrados dulceacuícolas presentes en el Parque Nacional Yendegaia. Valores del análisis de porcentajes de similitud (SIMPER) de la composición del ensamble de macroinvertebrados dulceacuícolas en ríos pluvio-nivales del Parque Nacional Yendegaia, sur de Chile. El porcentaje de corte de contribución se fijó en $80 \%$ de la abundancia del ensamble. Los datos están estandarizados y transformados a raíz cuadrada. 


\section{DISCUSIÓN}

Las condiciones naturales y de mínima intervención humana en los cuerpos de agua en el PN Yendegaia han permitido que las comunidades de macroinvertebrados dulceacuícolas permanezcan escasamente alteradas. La condición ecológica cuasi prístina de estos ríos, podría ser utilizada como referencia y con potencial bioindicador de las respuestas de la biodiversidad subantártica al estrés ambiental en el contexto de cambios ambientales globales. De la misma manera, al aumentar la conectividad terrestre y la visitación humana, se podría esperar que efectos sobre la vegetación ribereña se traduzcan en cambios estructurales en las comunidades de macroinvertebrados (Simanonok et al. 2011). La vegetación ribereña ofrece condiciones ambientales particulares (e.g. aporte de materia orgánica, diversidad de sustratos, temperatura, formación y duración de hielo superficial), que configuran, en mayor o menor grado, la estructura comunitaria de los macroinvertebrados dulceacuícolas (Contador et al. 2015, Simanonok et al. op. cit.).

La temperatura del agua y su variación en distintas escalas espaciales, es uno de los factores clave que influye directamente la distribución, ciclos de vida y fisiología de los macroinvertebrados dulceacuícolas (Vannote et al. 1980, Contador et al. 2016, 2020, Rendoll-Cárcamo et al. 2020b). No obstante, este no es el único parámetro, que puede influenciar la composición estructural de una comunidad bentónica. Los resultados aquí presentados, sugieren que la interacción de temperatura, $\mathrm{pH}$, conductividad, profundidad $\mathrm{y}$ presencia de macrófitas explicarían la variación entre las comunidades que co-habitan cada río. La presencia de macrófitas podría representar refugios microclimáticos y alimento para los macroinvertebrados en la misma forma que ciertas briófitas (Suren \& Winterbourn, 1992). Sin embargo, es necesario realizar estudios con un mayor esfuerzo espacial y temporal para evaluar este componente y las relaciones que pudiesen tener con las comunidades de macroinvertebrados. Las mediciones de $\mathrm{pH}$ para estos cursos de agua se mantienen circum-neutrales, concordantes con registros en varios ríos y arroyos de la Reserva de la Biosfera Cabo de Hornos (RBCH) (Tabla 1,
Moorman et al. 2006, Contador et al. 2015). De la misma manera, los valores de conductividad y sólidos disueltos totales sugieren una mínima cantidad de sales y minerales inorgánicos en estos y otros sistemas dulceacuícolas de la $\mathrm{RBCH}$ (Moorman et al. 2006, Mach et al. 2016). Las variaciones observadas en la temperatura entre los ríos podrían responder a factores no considerados en el estudio, como la cobertura arbórea, la distribución espacial y edades de la vegetación ribereña.

La riqueza de macroinvertebrados registrada fue similar entre los ríos con vegetación caducifolia (Nirre y Lenga), mientras que en siempreverde (Coihüe), esta fue significativamente más baja (Fig. 2). Factores como la estacionalidad podrían enmascarar la riqueza observada durante otoño de 2017 en estos ríos, encontrándose algunos macroinvertebrados en estadios tempranos de desarrollo (RendollCárcamo, 2018). La vegetación no sólo representa las condiciones físicas adyacentes, también constituye la fuente de recursos alóctonos de la que varios macroinvertebrados dependen (Anderson et al. 2018). No obstante, se hace necesario evaluar con mejor resolución las características funcionales de estas comunidades para poder explorar diferencias entre condiciones. Los órdenes de macroinvertebrados con mayor riqueza en los ríos de este parque nacional fueron Diptera y Trichoptera, patrón que se repite en cuerpos de agua en Isla Navarino y Tierra del Fuego (Simanonok et al. 2011, Contador et al. 2015, Anderson et al. 2018). Los patrones de abundancia de macroinvertebrados en estos ríos, aun cuando difieren entre sí, son similares a registros en Patagonia (Miserendino \& Pizzolón, 2000, 2003) y Andes Argentinos (Scheibler et al. 2014). Estas diferencias en las métricas de diversidad se traducen en diferentes estructuras comunitarias (Fig. 3), que pueden estar vinculadas a más de un factor gobernante (e.g. temperatura, disponibilidad de hábitat y nutrientes). Por ejemplo, los gradientes de elevación actúan como proxies de variables ambientales como la temperatura y reflejan cambios en la composición, estructura, y función de las comunidades bentónicas (Rendoll-Cárcamo et al. 2019). Si bien, en los ríos estudiados no se exploró el efecto de la elevación sobre la diversidad dulceacuícola de macroinvertebrados, la conjunción de factores abióticos (componentes del microhábitat) entregan 
información relevante sobre su distribución en escalas pequeñas. Los grupos representativos de estos ecosistemas, principalmente baétidos y leptoflébidos (Ephemeroptera), gripopterígidos (Plecoptera), limnefílidos (Trichoptera) y quironómidos (Diptera), presentan taxa comunes a otras áreas ya estudiadas de la RBCH (Moorman et al. 2006, Contador et al. 2015, Rendoll-Cárcamo et al. 2019). Cabe destacar, que en los ríos estudiados no se registraron dytíscidos o élmidos (Coleoptera), a diferencia de algunos estudios en la Isla Navarino (Contador et al. 2015). En cuanto a la estructura de las comunidades de macroinvertebrados en ríos del PN Yendegia, éstas presentan altos valores de equitatividad (indice de Pielou, Tabla 3), a excepción del río Nirre.

El presente trabajo refleja la necesidad de implementar, y complementar inventarios y estrategias de monitoreo en los sistemas dulceacuícolas de la $\mathrm{RBCH}$. Cabe destacar que estos ecosistemas australes representan el límite latitudinal de varios órdenes de insectos, como Ephemeroptera, Plecoptera y Trichoptera (Moorman et al. 2006, Rendoll-Cárcamo et al. 2019), sugiriendo una diversidad con alto nivel de endemismo (Valdovinos et al. 2010). El escaso impacto antropogénico en los ecosistemas dulceacuícolas de la $\mathrm{RBCH}$ permite evaluar el efecto de la variación ambiental natural sobre poblaciones y comunidades de macroinvertebrados, reduciendo el número de efectos confundidos (RendollCárcamo et al. 2020b). No obstante, este estudio tuvo lugar en otoño de 2017 y en sitios accesibles, por lo que una óptima caracterización biótica y abiótica debería incluir una escala espacio-temporal más amplia. El desarrollo e implementación de inventarios, monitoreo y estudio de patrones ecológicos permitirá avanzar en el conocimiento de la biodiversidad en estos australes y remotos ambientes dulceacuícolas, y establecer una línea base referencial en estos sitios, que han sido reconocidos para valoración SESELP (Sitios de Estudios Socio-Ecológicos a Largo Plazo).

\section{AGRADECIMIENTOS}

Los autores agradecemos a los revisores por sus sugerencias y comentarios que han ayudado enormemente a mejorar este manuscrito. Además, agradecemos el apoyo del Instituto de
Ecología y Biodiversidad (IEB, Financiamiento Basal ANID-AFB170008), al equipo del Programa de Conservación Biocultural Subantártica por su apoyo en las actividades de terreno. Peter Convey agradece el apoyo de NERC core funding al equipo "Biodiversity, evolution and adaptations" del Bristish Antarctic Survey (BAS). Este trabajo es una contribución del Laboratorio Wankara de Ecosistemas Dulceacuícolas Antárticos y subantárticos, y del Programa de Conservación Biocultural Subantártica.

\section{LITERATURA CITADA}

Anderson, C. B., Johnson, M., \& Lopez, M. E. (2018). Establishing habitat-specific indicator species in Tierra del Fuego with freshwater macroinvertebrates. New Zealand Journal of Marine and Freshwater Research, 52(1), 145-154.

Anderson, M. J. (2001). A new method for nonparametric multivariate analysis of variance. Austral Ecology, 26, 32-46.

Anderson, M. J. (2005). PERMANOVA: a FORTRAN computer program for permutational multivariate analysis of variance. Department of Statistics, University of Auckland, New Zealand, 24.

Anderson, M. J., Gorley, R. N., Clarke, K. R. (2008). PERMANOVA+ for PRIMER: guide to software and statistical methods. Plymouth, UK: PRIMER-E Ltd

Chao, A., Gotelli, N. J., Hsieh, T. C., Sander, E. L., Ma, K. H., Colwell, R. K., \& Ellison, A. M. (2014). Rarefaction and extrapolation with Hill numbers: a framework for sampling and estimation in species diversity studies. Ecological monographs, 84(1), 45-67.

Clarke, K. R. (1993). Non-parametric multivariate analysis of changes in community structure. Australian Journal of Ecology, 18, 117143.

Clarke, K. R. \& Gorley, R. N. (2015). PRIMER v7: User Manual/Tutorial. First edit.; Plymouth: Auckland, New Zealand.

Collen, B., Böhm, M., Kemp, R., \& Baillie, J. E. (2012). Spineless: status and trends of the world's invertebrates. United Kingdom: Zoological Society of London. 
Contador, T., Gañan, M., Bizama, G., FuentesJaque, G., Morales, L., Rendoll-Cárcamo, J., Simoes, F., Kennedy, J. Rozzi, R. \& Convey, P. (2020). Assessing distribution shifts and ecophysiological characteristics of the only Antarctic winged midge under climate change scenarios. Scientific Reports, 10(1), 1-12.

Contador, T., Kennedy, J. H., \& Rozzi, R. (2012), The conservation status of South American aquatic insects in the literature. Biodiversity and Conservation, 21, 2095-2107.

Contador, T., Kennedy, J. H., Rozzi, R. \& Ojeda, J. (2015), Sharp altitudinal gradients in Magellanic Sub-Antarctic streams: patterns along a fluvial system in the Cape Horn Biosphere Reserve (55 S). Polar Biology, 38(11), 1853-1866.

Domínguez, E. \& Fernández, H. R. (2009). Macroinvertebrados bentónicos sudamericanos. Sistemática y Biología. Fundación Miguel Lillo, Tucumán, Argentina.

Dudgeon, D. (2019). Multiple threats imperil freshwater biodiversity in the Anthropocene. Current Biology, 29(19), 960-967.

Flint, O. S., Holzenthal, R. W. \& Harris, S. C. (1999). Nomenclatural and Systematic changes in the Neotropical caddisflies (Insecta: Trichoptera). Insecta Mundi, 13, 73-84.

Hallmann, C. A., Sorg, M., Jongejans, E., Siepel, H., Hofland, N., Schwan, H., Stenmans, W., Müller, A., Sumser, H., Hörren, T., Goulson, D. \& de Kroon, H. (2017). More than 75 percent decline over 27 years in total flying insect biomass in protected areas. PLoS ONE, 12(10), e0185809.

Hedin, L. O., Armesto, J. J. \& Johnson, A. H. (1995). Patterns of nutrient loss from unpolluted, old-growth temperate forests: Evaluation of biogeochemical theory. Ecology, 76(2), 493-509.

Mach, P. M., Winfield, J. L., Aguilar, R. A., Wright, K. C., \& Verbeck, G. F. (2017). A portable mass spectrometer study targeting anthropogenic contaminants in Sub-Antarctic Puerto Williams, Chile. International Journal of Mass Spectrometry, 422, 148-153.
Mancilla, G., Valdovinos, C., Azocar, M., Jorquera, P., \& Figueroa, R. (2009). Efecto del reemplazo de la vegetación nativa de ribera sobre la comunidad de macroinvertebrados bentónicos en arroyos de climas templados, Chile central. Hidrobiológica, 19(3), 193203.

McLellan, I. D. \& Swick, P. (2007). New species and keys to South American Gripopterygidae (Plecoptera). Illiesia, 3(4), 20-42.

Merritt, R. W., \& Cummins, K. (2008). An introduction to the aquatic insects of North America. Dubuque, IA: Kendall/ Hunt Publishing Company.

Miserendino M. L. \& Pizzolón L. A. (2000). Macroinvertebrates of a fluvial system in Patagonia: altitudinal zonation and functional structure. Archiv Fur Hydrobiologie, 150, 55-83

Miserendino M. L. \& Pizzolón L. A. (2003). Distribution of macroinvertebrate assemblages in the Azul-Quemquemtreu River Basin, Patagonia, Argentina. New Zealand Journal of Marine and Freshwater Research, 37, 525-539.

Moorman M. C., Anderson C. B., Gutiérrez A. G., Charlin R. \& Rozzi R. (2006). Watershed conservation and aquatic benthic macroinvertebrate diversity in the Alberto D’Agostini National Park, Tierra del Fuego, Chile. Anales del Instituto de la Patagonia, 34, 41-58.

Morrone, J. J. (2015). Biogeographical regionalisation of the Andean region. Zootaxa, 3936(2), 207-236.

Reid, A. J., Carlson, A. K., Creed, I. F., Eliason, E. J., Gell, P. A., Johnson, P. T., Kidd, K. A., MacCormack, T. J., Olsen, J. D., Ormerod J. D., Smol, J. P., Taylor, W. W., Tockner, K., Vermaire, J. C., Dudgeon, D., \& Cooker, S. J. (2019). Emerging threats and persistent conservation challenges for freshwater biodiversity. Biological Reviews, 94(3), 849-873.

Rendoll-Cárcamo, J. (2018). Variación estacional y altitudinal en la diversidad, distribución y tolerancias térmicas de macroinvertebrados dulceacuicolas sub-Antárticos: en la búsqueda de 
bioindicadores térmicos para el cambio climático global. Tesis de Magíster. Punta Arenas, Chile: Departamento de Recursos Naturales, Facultad de Ciencias, Universidad de Magallanes.

Rendoll-Cárcamo, J., Contador, T., Gañán, M., Houston, N., Troncoso, M., Arriagada, G., Saldías, C., Caballero, P., Malebrán, J., Kennedy, J., Convey, P. \& Rozzi R. (2020a). Filosofía Ambiental de Campo: Educación e investigación para la valoración ecológica y ética de los insectos dulceacuícolas. Magallania, 48(2), 213-228.

Rendoll-Cárcamo, J., Contador, T., Convey, P. \& Kennedy, J. (2020b). Sub-Antarctic Freshwater Invertebrate Thermal Tolerances: An Assessment of Critical Thermal Limits and Behavioral Responses. Insects, 11, 102.

Rendoll-Cárcamo, J., Contador, T., Gañán, M., Troncoso, C. P., Márquez, A. M., Convey, P., Kennedy, J. \& Rozzi, R. (2019). Altitudinal gradients in Magellanic subAntarctic lagoons: the effect of elevation on freshwater macroinvertebrate diversity and distribution. PeerJ, 7, e7128.

Rozzi, R., Armesto, J.J., Gutiérrez, J., Massardo, F., Likens, G., Anderson, C.B., Poole, A., Moses, K., Hargrove, G., Mansilla, A., Kennedy, J.H., Wilsson, M., Jax, K., Jones, C., Callicott, J.B. \& Kalin, M.T. (2012). Integrating ecology and environmental ethics: Earth stewardship in the southern end of the Americas. BioScience, 62(3), 226-236.

Rozzi, R., Massardo, F., Berghoefer, A., Anderson, C. B., Mansilla, A., Mansilla, M., Plana, J., Berghoefer, U., Araya, P. \& Barros, E. (2006). The Cape Horn Biosphere Reserve, Punta Arenas, Chile: Ediciones Universidad de Magallanes.

Sánchez-Bayo, F., \& Wyckhuys, K. A. (2019). Worldwide decline of the entomofauna: A review of its drivers. Biological conservation, 232, 8-27.

Scheibler, E. E., Claps, M. C. \& Roig-Juñent, S. A. (2014). Temporal and altitudinal variations in benthic macroinvertebrate assemblages in an Andean river basin of Argentina. Journal of Limnology, 73(1), 92-108.

Servicio Nacional de Turismo (2014). Plan de acción Región de Magallanes y Antártica Chilena Sector Turismo 2014-2018. Sernatur, Santiago de Chile.

Schüttler, E., Crego, R. D., Saavedra-Aracena, L., Silva-Rodríguez, E. A., Rozzi, R., Soto, N., \& Jiménez, J. E. (2019). New records of invasive mammals from the sub-Antarctic Cape Horn Archipelago. Polar Biology, 42(6), 1093-1105.

Simanonok, M. P., Anderson, C. B., Pastur, G. M., Lencinas, M. V., \& Kennedy, J. H. (2011). A comparison of impacts from silviculture practices and North American beaver invasion on stream benthic macroinvertebrate community structure and function in Nothofagus forests of Tierra del Fuego. Forest Ecology and Management, 262(2), 263-269.

Suren, A. M. \& Winterbourn, M. J. (1992). The influence of periphyton, detritus and shelter on invertebrate colonization of aquatic bryophytes. Freshwater Biology, 27(3), 327-339.

Valdovinos, C., Kiessling, A., Mardones, M., Moya, C., Oyanedel, A., Salvo, J., Olmos, V. \& Parra, Ó. (2010). Distribución de macroinvertebrados (Plecoptera y Aeglidae) en ecosistemas fluviales de la Patagonia chilena: ¿muestran señales biológicas de la evolución geomorfológica postglacial?. Revista Chilena de Historia Natural, 83(2), 267-287.

Vannote, R. L., Minshall, G. W., Cummins, K. W., Sedell, J. R. \& Cushing, C. E. (1980). The river continuum concept. Canadian journal of fisheries and aquatic sciences, 37(1), 130-137.

Vörösmarty C. J., McIntyre P. B., Gessner M. O., Dudgeon D., Prusevich A., Green P., Glidden S., Bunn S. E., Sullivan C. A., Reidy Liermann C. \& Davies P. M. (2010). Global threats to human water security and river biodiversity. Nature, 467, 555-561. 